\title{
Global Longitudinal Strain as a Predictor of Chemotherapy-Induced Cardiotoxicity
}

\author{
Monica Avila ${ }^{1}$, Marco Alves², Silvia Ayub-Ferreira ${ }^{2}$, Mauro Wanderley Junior ${ }^{2}$, Fatima \\ Cruz $^{2}$, Sara Michelly Brandão ${ }^{2}$, Ludhmila Hajjar ${ }^{2}$, Roberto Kalil Filho ${ }^{2}$, Cecilia Cruz ${ }^{3}$, M. \\ Cristina Abduch ${ }^{4}$, Danilo Moleta ${ }^{2}$, and Edimar Bocchi ${ }^{2}$ \\ ${ }^{1}$ University of Sao Paulo Heart Institute \\ ${ }^{2}$ Affiliation not available \\ ${ }^{3}$ Heart Institute/University of São Paulo \\ ${ }^{4}$ University of Chicago
}

May 5, 2021

\begin{abstract}
Background: Chemotherapy-induced cardiotoxicity (ChC) is an important complication among patients receiving anthracyclines. Biomarkers and imaging parameters have been studied for their ability to identify patients at risk of developing this complication. Left ventricle global longitudinal strain (LV-GLS) has been described as a sensitive parameter for detecting systolic dysfunction, even in the presence of preserved left ventricle ejection fraction (LVEF). Objective: to evaluate the role of the LV-GLS as a predictor of ChC. Methods: This study is a post-hoc analysis of CECCY trial (Carvedilol for Prevention of Chemotherapy-Related Cardiotoxicity) that evaluated the primary prevention of cardiotoxicity with carvedilol during doxorubicin chemotherapy in a population with breast cancer. Cardiotoxicity was defined as a reduction >10\% in LVEF. LV-GLS was obtained before chemotherapy in patients with no prior cardiovascular disease or echocardiogram abnormalities. Results: Thirty-one patients who had a complete echocardiography study including measurement of LV-GLS before chemotherapy were included in this analysis. An absolute LV-GLS $<16.9 \%$ before chemotherapy showed $100 \%$ sensitivity and $73 \%$ specificity for predicting cardiotoxicity ( $\mathrm{AUC}=0.85 ; 95 \% \mathrm{CI} 0.680-0.959, \mathrm{p}<0.001)$. In this population, LVEF values before chemotherapy did not predict $\mathrm{ChC}(95 \% \mathrm{CI} 0.478$ to $-0.842, \mathrm{p}=0.17)$. The association of low LV-GLS $(<17 \%)$ and BNP serum levels $(>17$ $\mathrm{pg} / \mathrm{mL}$ ) two months after chemotherapy increased the accuracy for detecting early onset ChC (100\% sensitivity, $88 \%$ specificity, $\mathrm{AUC}=0.94 ; 95 \%$ CI $0.781-0.995, \mathrm{p}<0.0001)$. Conclusions: Our data suggest that LV-GLS is a potential predictor of chemotherapy-induced cardiotoxicity. Larger studies are needed to confirm the relevance of this echocardiographic parameter in this clinical setting.
\end{abstract}

\section{Hosted file}

Manuscript file.pdf available at https://authorea.com/users/404908/articles/520817-globallongitudinal-strain-as-a-predictor-of-chemotherapy-induced-cardiotoxicity 
A
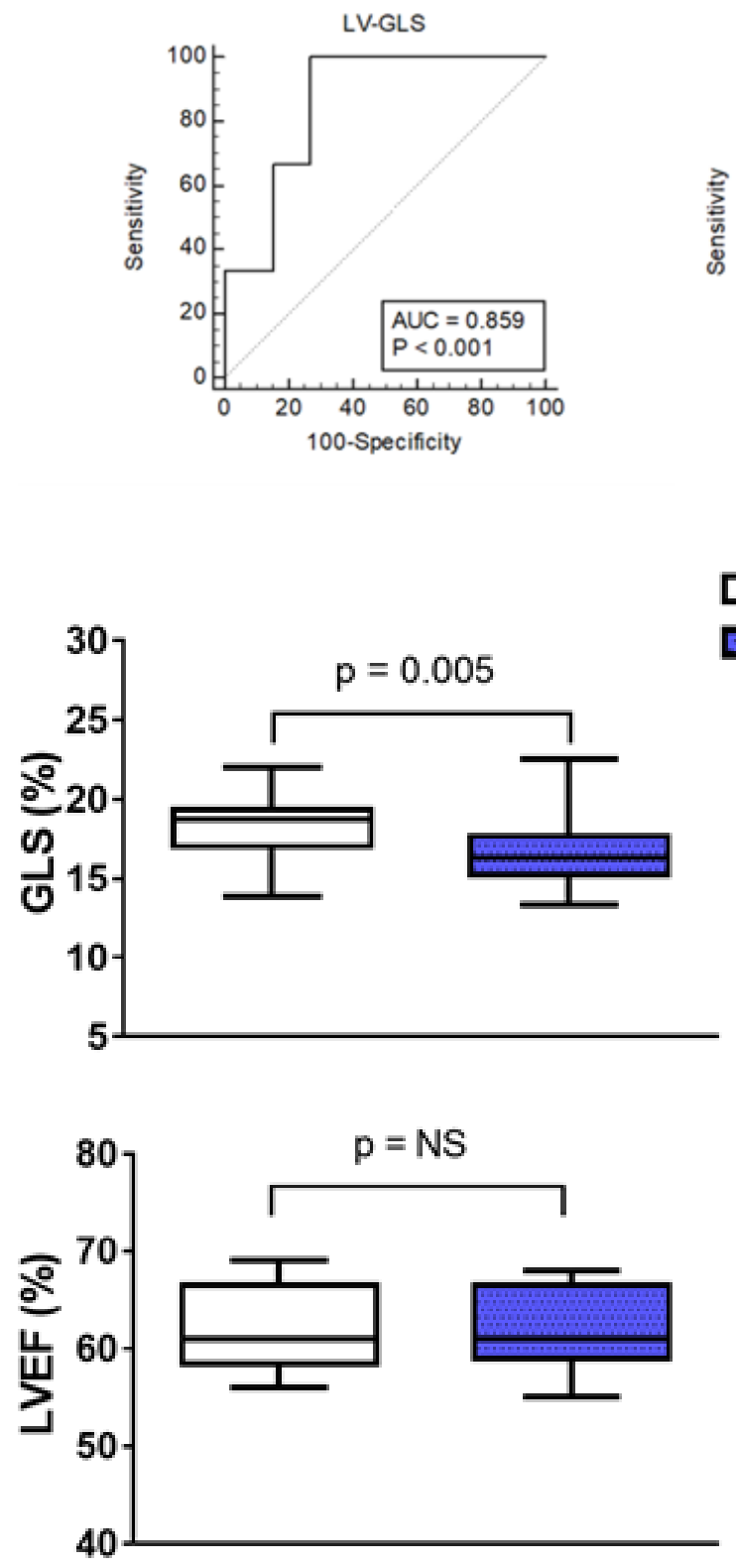

B

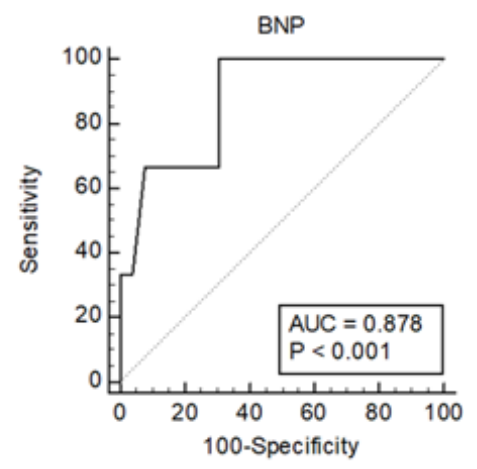

$\square$ Before ChT

12 months after ChT 